Diánoia, vol. 34, no. 34, 1988

textos filosóficos bastante frecuentes, textos pretendidamente de historia de la filosofía, pero en los cuales encontramos, junto con la presentación de las ideas de un autor del pasado, desarrollos originales de estas ideas por parte del historiador-filósofo. Lo que me parece ilegítimo de esta mezcla de filosofía e historia es que lleva a que ni se satisfagan criterios filosóficos de verdad, ni criterios históricos de veracidad u objetividad. En efecto, lo que usualmente sucede en este tipo de textos híbridos es que se considera que no hay que dar razones para probar la verdad de la idea o teoría desarrollada, ya que se está haciendo historia, pero precisamente esta falta de argumentación es la que da lugar a que la filosofía allí desarrollada sea mala filosofía; por otra parte, tampoco se considera que importe demasiado la falta de fidelidad histórica, ya que se le llega dar más interés al desarrollo de cierta idea o teoría del autor pasado que se estudia, falta de fidelidad que hace que la historiografía presentada en dichos textos híbridos sea una mala historia.

\title{
FILOSOFÍA E HISTORIA DE LA FILOSOFÍA
}

MaURicio Beuchot

Instituto de Investigaciones Filosóficas, UNAM

Las preguntas que orientan el siguiente trabajo son dos: (i) ¿Cuál es la importancia de la historia de la filosofía para hacer filosofía? $y$, en el supuesto de que la historia de la filosofía sea útil para hacer filosofía, (ii) ¿cuál es un buen método para hacer dicha historia de la filosofía? ${ }^{9}$

Frente a las polémicas acerca de si tenemos que acercarnos más a la recuperación del pasado o inclinarnos más a la comunicación del pasado de una manera relevante para los lectores del presente, me parece que una buena aportación metodológica nos la brinda la conjunción de hermenéutica y teoría de la argumentación. La hermenéutica nos enseña que siempre se introduce nuestra subjetividad cultural en la historia que leemos o que escribimos; por ello nuestra actitud historiográfica tiene limitaciones, y hay que dar un mayor énfasis al diálogo interpretativo con los filósofos de la historia pasada. Además, veo que en el diálogo con los autores del pasado vale lo que ocurre en el dialogo con los actuales: hay condiciones para

9 Sobre las posibles relaciones de la filosofía y la historia de la filosofía, cfr. J. J. E. Gracia, "La filosofía y su historia", en Revista Latinoamericana de Filosofía, 13 (1987), pp. 259-278. 
que el diálogo sea fructífero, y por eso me parece que al filósofo le ayuda sobre todo el atender a aquellos autores y corrientes que comparten en mayor medida sus principios y/o reglas que más se acercan a sus intereses, temas y métodos. La razón obvia de esto es que, sin un acuerdo básico sobre reglas y principios (tanto lógicos como ontológicos), el diálogo puede llegar a resultar imposible. Será en esa línea como obtendrá el mayor provecho de su diálogo con filósofos anteriores, al analizar sus planteamientos, sus respuestas y sus argumentos. Como se ve, entiendo la metodología de la historia de la filosofía más como un diálogo argumentativo con los filósofos que como inacabable historiografía; claro que exijo lo más que se pueda tomar en cuenta del contexto histórico y filológico, pero veo la historia de la filosofía preponderantemente como un diálogo con los filósofos antiguos en el que se examinan tesis y se evalúan argumentos para apoyarlas, de modo muy parecido al diálogo que mantenemos con los filósofos modernos.

La hermenéutica contemporánea, p. ej. en la teorización de P. Ricoeur, ${ }^{10}$ nos enseña que no existe una historia sin reconstrucción interpretativa, ni tampoco una historia que sea pura reconstrucción; hay un "estira y afloja" entre la historiografía objetivista y la interpretación en la que introducimos nuestra individualidad cultural. Los textos filosóficos ya no tienen los mismos destinatarios, tienen otros: los lectores modernos, que les hacen perder su autonomía original. Si no, no hay contacto con ellos. Por otra parte, no podemos hacer historia cambiando lo que quiso decir el autor. Por lo tanto, nos queda la abrumadora responsabilidad de no traicionar al autor. Es decir, nuestra hermenéutica consiste en recuperar la intencionalidad del autor y comunicarla al lector contemporáneo. Difícil equilibrio, en el que a la hermenéutica - que insiste más en el sentido que añade el lector o intérprete- le ayuda la pragmática - que insiste más en el sentido que quiso dar el autor. ${ }^{11}$ Hermeneutizar es conectar el texto con su contexto de época y a la vez conectarlo con el presente. Por lo general incurrimos en algún extremo: o somos más historiógrafos o somos más analíticos de argumentos. Inclusive me atrevería a decir que estamos más inclinados a ser menos historiógrafos por la distancia cultural. Pero también creo que, si ese riesgo es ineludible, lo mejor es sacar el mayor provecho del énfasis de la historia de la filosofía como diálogo con los filósofos anteriores "como si fueran presentes".

10 De la hermenéutica ricoeuriana me ocupo en mi trabajo Hermenéutica, lenguaje $e$ inconsciente, Universidad Autónoma de Puebla, Puebla (México), 1988.

11 Véase el artículo de Marcelo Dascal sobre hermenéutica y pragmática en Rivista de filosofia neoscolastica, fascículo 3 de 1988. 
Al estudiar la filosofía medieval al trasluz de la actual "filosofía analítica", yo me siento como en un diálogo contemporáneo y dentro de un debate interesante cuando comparo tesis y argumentos de Duns Escoto y Suárez con tesis y argumentos de David Wiggins y Michael Slotte en cuanto a la teoría ontológica de la substancia. Hay una continuidad, una especie de progreso que se da criticando y/o mejorando argumentos de los filósofos. Aunque las tesis son análogas, no son exactamente las mismas. Por ejemplo, la teoría de los nombres propios y la de la filosofía del lenguaje en general de Ockham se parece a la de Russell - como observa Geach- en muchos aspectos, mientras que la de G. de Sherwood se acerca a la de Frege y algo a la de Strawson; San Vicente Ferrer pelea con los ockhamistas e introduce tesis de tipo Quine. ${ }^{12}$ Pero no son lo mismo, y en ese no ser lo mismo nos enriquecen con otros caminos y argumentos. Un discípulo de Hintikka, S. Knuutila, dice que Sto. Tomás sería una especie de Kripke de la Edad Media, mientras que Escoto sería una especie de David Lewis de la misma, por la semejanza de sus posturas. Lo que cuenta, pues, son los debates con aquellos filósofos con los que podemos lograr alguna conmensuración. ${ }^{13}$

¿De qué sirve la historia de la filosofía para hacer filosofía? En esta ponencia me propongo argüir a favor de la tesis de que sí sirve al filósofo estudiar la historia de la filosofía para ponerse a filosofar. Sobre todo, claro está, al filósofo le ayuda atender en su filosofar a aquellas corrientes que más se acercan a sus intereses y participan más de sus temas y métodos.

En efecto, aun cuando en otras ramas del saber no ayuda mucho el estudiar la historia de esa disciplina en cuestión, en el caso de la filosofía es diferente; estudiar la historia de la filosofía ayuda a hacer filosofía, porque el avance en la tradición filosófica (incluso dentro de una tradición filosófica) es más cualitativo que cuantitativo en comparación con el que se da en las ciencias. Además, depende mucho menos que las ciencias de la contrastación empírica de las teorías y más de los argumentos especulativos que ofrezca.

Puede decirse que - a diferencia de las ciencias- en filosofía las más de las veces se avanza en intensión y no tanto en extensión, o que el mismo aumento extensivo redunda en el aumento intensivo o de profundización. Así, aunque para la física actual, en el campo de la mecánica, no dice mucho la teoría medieval del "impetus", en cambio en filosofía sí puede decirnos mucho una teoría medieval

12 Sobre estos tópicos, ver mi trabajo La filosofía del lenguaje en la Edad Media, Instituto de Investigaciones Filosóficas, México, UNAM, 1981.

13 Acerca de esto, véase C. Pereda, "Historia explicativa y lectura argumentada", en Diálogos (El Colegio de México), n. 128 (1985), pp. 19-24. 
o antigua de la substancia. Por ello el "progreso" en filosofía (si podemos llamarlo así) requiere más de la reflexión sobre lo ya visto, de la vuelta a lo ya obtenido y logrado. Esa vuelta sobre lo ya dado nos servirá para criticar y encontrar errores o para descubrir cosas valiosas en las reflexiones pretéritas, y así la atención a la historia de la filosofía resultará muy pertinente al filósofo. La filosofía requiere de mucha más reflexión, en el sentido original y etimológico de la palabra, "volver a doblar" o "redoblar" nuestros pensamientos sobre el asunto.

Dado este carácter reflexivo de la filosofía, nos vemos obligados a volver a pensar y repensar los problemas y las soluciones, en lo cual nos encontramos muchas veces con que nuestros compañeros de camino son pensadores de épocas ya pasadas, y el ser anterior en el tiempo no significa estar ya sin más superado, ni el ser posterior en el tiempo significa tampoco que la especulación es mejor o más certera que la de los anteriores. De hecho hay teorías, por ejemplo metafísicas, que no han sido superadas por los actuales en su fuerza y sofisticación, y todo por haber sido sencillamente relegadas al polvo de la historia; tal me parece que ocurre con la teoría de la substancia de Aristóteles, con la teoría de la esencia y la existencia de Tomás de Aquino, con la teoría de la individuación de Duns Escoto y con la teoría de las distinciones de Suárez. Compiten con las teorías alternativas que se han producido en la actualidad sobre esos temas. Eso nos muestra que en filosofía no se da un progreso temporal de modo simple, puede encontrarse el que un filósofo anterior haya planteado mejor los problemas o haya dado soluciones más atinadas, pero que nos ha faltado conocer sus doctrinas. $Y$ esas doctrinas son conmensurables y se comparan con las actuales y rivalizan con ellas dependiendo de quién ofrezca mejores argumentos. ${ }^{14}$

En filosofía sí se corre el riesgo de repetir - por desconocimiento debido a la cerrazón en atender a su historia- errores ya dados, se corre también el riesgo de repetir mal los aciertos que otros han tenido, en lugar de mejorarlos. Pues incluso hay la posibilidad de que un autor lejano al presente tenga una solución mejor que la que se ofrece hoy en día a determinado problema. Se podrá decir que la historia de la filosofía sólo servirá en muchos casos para constatar que en realidad no ha habido ninguna teoría mejor que la que hoy se profesa, pero aún así ya tiene sentido estudiar la historia de la filosofía. Claro que esto no ocurre así, de una manera tan directa

14 Un poco más adelante hablaremos de la conmensurabilidad entre teorías filosóficas y también acerca de las condiciones que se pueden requerir para que haya diálogo entre ellas y operen o funcionen los argumentos que en dicho diálogo se aduzcan. 
y ramplona, o de una manera tan drástica como vemos que ocurre en la ciencia, es decir en las tradiciones científicas. Esto sucede sobre todo porque en filosofía hay menos dependencia de los datos empíricos, una teoría filosófica no se puede criticar, rechazar o mejorar, sólo por un conocimiento de los datos cuantitativamente más amplio que el que se tenía antes, sino que el avance o la mejoría dependen de que se comprendan intelectivamente de mejor manera. Un pensador con pocos instrumentos empíricos pudo haber tenido una intelección mejor que la que tenemos ahora con mucho material empírico, ya que en filosofía lo primario es la calidad de la intelección con la cual penetramos un problema y sus datos, lo secundario es la cantidad de los datos o experimentos con los que pretendemos apoyar la teoría. Pues la intelección puede darse con muy pocos experimentos y, a la inversa, pueden tenerse muchos experimentos y no ocurrir la intelección. En todo caso, es algo difícil (casi paradójico) decir cuántos experimentos o datos justifican la intelección que tuvimos. Casi tenemos que decir que es algo intuitivo y hasta prudencial. ${ }^{15}$

En filosofía no es a priori verdadera la postura "sublacionista" o "superacionalista" de algunos pretendidos hegelianos, ${ }^{16}$ con su Aufhebung, según la cual todo pensamiento anterior está ya superado (aufgehoben) por el posterior. Eso es darle al paso del tiempo un toque mágico con el cual, por el simple hecho de ser nuevo, es mejor. Claro, tampoco por el hecho de ser anterior es verdadero, como si hiciéramos valer el verso de Jorge Manrique, según el cual "todo tiempo pasado fue mejor", sino que están en igualdad de condiciones en principio. Y todo dependerá de quién aporte los argumentos más convincentes, que mejor persuadan de lo que se sostiene. No hay, pues, "sublacionismo" o "superacionismo" en la historia de la filosofía. No podemos decir que algo está superado sin más sólo por ser anterior. No es el tiempo o la cronología lo que hace superar un pensamiento, sino los argumentos, ya apodícticos, ya tópicos, ya retóricos.

Hablamos de argumentar entre pensadores de la historia de la filosofía como si fueran contemporáneos que dialogaran, pero ciertamente este diálogo y esta argumentación tienen sus requisitos de contextuación y de pertinencia. No se trata sólo de una historia de la filosofía hecha en signo filológico, sino también en signo analíticoargumentativo, en la que se atiende sobre todo a los argumentos de

Es el mismo caso de la inducción, que nos remite a la paradoja del sorites (o sorós, "montón" en griego), según la cual se nos pregunta si un grano de arena constituye un montón, o dos granos, o tres, o cuatro, etc.

${ }^{16}$ Cfr. W. Redmond, "Filosofía tradicional y pensamiento latinoamericano. Superación y vigencia”, en Prometeo (U. de G.-CCyDEL), n. 2 (1985), pp. 43-57. 
los filósofos como en un diálogo. Acerquémonos, pues, un tanto, al examen de las condiciones para que se dé esa posibilidad dialógica.

Lo más frecuente es que encontremos entre los filósofos del pasado alguno o algunos con los que entramos en diálogo de manera preferente. ${ }^{17}$ Es raro que alguien tenga la ingente capacidad de dialogar profundamente con todos los que registran las historias; más usual es que encuentre alguno o unos cuantos como objeto de su predilección, en los que se especializa y con los que mantiene una actitud de escucha o de aprendizaje, y a los que igualmente critica. En realidad, es un diálogo variado y rico el que mantiene con ellos.

Alasdair MacIntyre sostiene que no podemos decir qué modelo de racionalidad es el mejor; y su argumento consiste en decir que eso implicaría que tenemos algún criterio meta-racional y metahistórico o el meta-criterio de la racionalidad por encima de nuestra propia racionalidad concreta que nos permitiría medir y enjuiciar los demás modelos racionales. ${ }^{18}$ Pero, como sabemos, ante ello sólo podemos bajar la cabeza y admitir que en verdad no tenemos ese meta-criterio que atraviesa y cubre meta-filosóficamente o diafilosóficamente toda la historia de la filosofía. Sin embargo, hay algo que se acerca a ese meta-criterio y vale solamente para una tradición filosófica. Por ejemplo, aunque no todas las escuelas filosóficas aceptan la lógica formal y sus principios, si uno admite eso, se sentirá más inclinado a estudiar la historia de esas escuelas filosóficas que sí lo hacen, y a sacar el mayor provecho de esos que reflexionaron siguiendo esos cánones metodológicos. En esa línea de compartir la misma tradición, es indudable que se obtiene el mayor provecho. Se incrementan los recursos teóricos y son más directas las objeciones y mayor la posibilidad de discutir argumentos, porque se comparten los mismos principios. Se llenan de la mejor manera las condiciones para el diálogo. $\mathrm{Y}$, conforme van siendo menos los pricipios que se comparten, se va perdiendo lo fructífero de la discusión, llegando al punto en que se comete petición de principio dialógica, a saber, de argumentar por lo que el otro no cree ni acepta. Y entonces ¿cómo discutir entre escuelas o tradiciones distintas?

Cuando nos aproximamos a los autores y escuelas filosóficos del pasado no sólo para repetir lo que dicen sino para beneficiarnos de

$17 \mathrm{Y}$ esto puede verse en la actualidad; se dialoga con provecho por cierta "afinidad de espíritu", no únicamente por cercanía cronológica. Cuántas veces un pensador de nuestra época prefiere dialogar con uno del pasado que con un contemporáneo suyo de diferente signo. Al parecer, nos une más esa afinidad de escuela, de método, de intereses, etc., que la mèra cercanía en el tiempo.

18 Ver A. MacIntyre, "The Relationship of Philosophy to its Past", en R. Rorty, J. B. Schneewind y Q. Skinner (eds.), Philosophy in History, Cambridge University Press, Cambridge, 1984, pp. 35 ss. 
su aportación aceptándola o combatiéndola, nos ocurre lo mismo que hoy en día: hemos de buscar los puntos de conmensuración. La aplicación de Kuhn por parte de MacIntyre a nuestro tema nos hace ver que, si en la historia de la ciencia las teorías han sido inconmensurables por no poder ser referidas a la base empírica que decida su verdad o falsedad, en el caso de las teorías filosóficas es mucho mayor el problema de su conmensurabilidad, ya que es más lejana la contrastación empírica. ${ }^{19} \mathrm{Y}$, por ello, la comparación entre teorías alternas se centra más: en el aspecto de su estructuración interna. Mas para optar por alguna de ellas o para rechazarla, esto es, para dialogar con ella, tenemos que cumplir las exigencias del diálogo, entre las cuales se encuentra en los primeros lugares la de compartir los mismos principios. De otra manera el "diálogo" no existirá y no: sacaremos ningún fruto.

Y es que la historia de la filosofía puede entenderse como un. diálogo con los autores del pasado; pero el diálogo auténtico y fructífero; con los autores del pasadio (como con los del presente) tiene siempre dos funciones: aceptar o refutar. A veces se acepta todo lo que dice un autor; otras veces se rechaza todo; pero lo más frecuente es que aceptemos. algunas cosas y rechacemos otras. Pero, como hemos dicho, sólo: podemos argumentar demostrativamente (de manera apodíctica o de manera tópica) lo que aceptemos o lo que rechacemos si compartimos los mismos principios. ${ }^{20}$ Cuando no se comparten, la argumentación se volverá imposible, o tendrá sólo un resquicio, a saber, se volverá retórica. Sólo habrá persuasión, no demostración.

Pero hay grados en ese compartir los mismos principios, y de esa manera resulta que podemos acercarnos a, o alejarnos de, un diálogo también según grados. Hay un espacio de diálogo con las diferentes escuelas filosóficas, desde las que pertenecen a nuestra misma tradición, hasta las que tienen principios tan distintos que ya no permiten una argumentación directa. Vale la pena conocer lo más posible la historia de la filosofía; pero, dadas las exigencias del diálogo y de la argumentación válida, por ello hemos dicho que sobre todo resulta fructífero dialogar con las corrientes, escuelas y pensadores que más se acercan a la tradición que compartimos. Y es que, ¿de qué nos valemos y en qué sostenemos nuestras tesis cuando no hay los mismos principios y reglas? Incluso se puede llegar a decir que no solamente hacen falta los mismos principios lógico-metodológicos (p. ej. el de no contradicción -en alguno de sus grados o formulaciones-, que

19 Ver A. MacIntyre, art. cit., pp. 41 ss.

20 Sobre esto puede verse "Acerca de la posibilidad del diálogo" en M. Beuchot y E. González Ruiz, Ensayos sobre teoría de la argumentación, UAM, México, en prensa. 
permitiría una - por lo menos - lógica formal; o el de tercio excluso, que la constriñe más, etc.), sino hasta algunos principios metafísicos u ontológicos; pues, a quien no acepta más que un mundo empirista en el sentido de fisicalista, ¿cómo le podemos argumentar a favor de la existencia de lo espiritual? Parece haber sólo un gran impasse.

En conclusión, dentro de ciertos límites que imponen las condiciones y requisitos de todo diálogo, se puede dialogar fructíferamente con los filósofos del pasado del mismo modo que con los del presente; $y$, si no queremos enconcharnos encerrados en nuestro propio discurso, si para hacer filosofía necesitamos del diálogo con otros pensadores, podemos entablarlo tanto con nuestros contemporáneos como con los que nos han precedido. Las condiciones del diálogo son las mismas para unos y otros; no se justifica a priori la sola preferencia por el diálogo con los del presente; resulta igualmente fructífero el diálogo con los del pasado, máxime si se ubican en una misma tradición y comparten principios parecidos a los nuestros, según grados. Nos ayudarán a probar o disprobar nuestras teorías, a sostener su plausibilidad dentro de un sistema o contexto más o menos común. Si salimos de ese contexto, al' iguall que: nos sucede con los actuales, hemos de recurrir a otro tipo de metodología, la que dimos en llamar "retórica" o persuasiva, siempre dentro de una perspectiva en la que lo que vale y nos avala son los argumentos que ofrezcamos ( $y$; como vimos, la argumentación, al igual que el diálogo, tiene sus: requisitos):. Pues bien, en esta misma línea dialógica frente a la historia de la filosofía, es decir, de acercarse a la historia de la filosofía para dialogar con ella, para argumentar con los pensadores del pasado que pueden ofrecernos algo más aprovechable para nuestro filosofar, se sitúa la mejor ayuda que ellos y la historia de la filosofía pueden brindarnos, y es la de movernos a la autocrítica.. ${ }^{21}$

21. Para entender la historia de la filosofía como una historia de argumentos que aceptamos o rechazamos, es necesario introducir el enfoque hermenéutico y pragmático, por el cual entendemos lo que el pensador quiere decir en sus tesis y argumentaciones. Sobre esta necesidad de la hermenéutica hemos hablado en M. Beuchot, "Hermenéutica de la historia de la filosofía en el México, de la colonia”, en Prometeo (U. de G.-CCyDEL), n. 10 (1987); pp. 78-88; sobre la pertinencia de la pragmática, que nos: oblïga a atender al menos en cierta medida a la historia "externa" o "externalista" de: la filosofía, hemos hablado en idem; "Hacia una metodología de la historia de la filosofía en el México colonial", en J. J. Saldaña (comp.), Ier. Simposio Mexicano de Historia de la Ciencia y la Tecnología, México, 1988, en prensa. 